\title{
NOUVELLE
}

\section{Stéroïdes, insuline et croissance Les mouches dopent la recherche}

Julien Colombani, Laurence Bianchini, Sophie Layalle, Pierre Léopold
Institut de Signalisation, Biologie du Développement et Cancer (ISBDC), CNRS UMR 6543 ,

Parc Valrose, 06108 Nice Cedex 2, France. leopold@unice.fr

J. Colombani (adresse actuelle):

Cancer Research UK, London Institute, 44 Lincoln's Inn Fields,

Londres WC2A 3PX, Royaume Uni. proportions harmonieuses qui caractérisent l'espèce en adaptation avec les conditions environnementales [1]. Au sein d'un organisme, la croissance d'un organe fait appel à trois processus: la croissance cellulaire qui augmente la taille des cellules, la prolifération cellulaire et la mort cellulaire qui régulent le nombre de cellules. La taille et la forme finales d'un organe sont régulées à la fois par des signaux extrinsèques de type hormonaux et par le programme génétique dépendant de l'activité des morphogènes $[2,3]$. Deux paramètres principaux déterminent l'étendue de la croissance tissulaire: la vitesse de croissance des cellules et la durée de la période de croissance. Chez la plupart des organismes supérieurs, la période de croissance est restreinte aux phases juvéniles du développement et s'achève par une étape de maturation sexuelle. Deux types distincts de signaux hormonaux modulent ces paramètres. Les molécules de la famille des insulines régulent le rythme de la croissance, alors que les transitions développementales permettant d'atteindre la maturation sont sous le contrôle d'hormones stéroïdiennes. Le lien entre ces deux systèmes qui déterminent la taille finale d'un organisme est largement inconnu. La drosophile s'est imposée au fil des dernières années comme un excellent modèle d'étude de la croissance. Chez cet insecte dit «holométabole» car il subit une métamorphose, les périodes embryonnaires et larvaires se succèdent et conduisent à la formation d'une pupe au sein de laquelle se reconstruisent les futurs tissus adultes. Du fait de la métamorphose pupale, la taille finale de l'animal est déterminée par la taille de la larve à l'issue d'une période de forte croissance larvaire (Figure $1 A$ ) $[4,5]$. De manière comparable à la situation des vertébrés, la vitesse de croissance chez la larve de drosophile est stimulée par des molécules de la famille des insulines, appelées Dilp (pour drosophila insulin-like peptides), tandis que la fin de la période de croissance larvaire est sous le contrôle de l'ecdysone, la principale hormone stéroïdienne chez les insectes. La signalisation insuline/ IGF (IIS) a été particulièrement étudiée pour son implication dans le contrôle du taux de croissance cellulaire in vivo. Chez les invertébrés, I'IIS est fortement conservée et les Dilp portent à la fois les fonctions métaboliques de l'insuline et les fonctions de croissance des IGF [6]. Ces molécules sont sécrétées par deux groupes de neurones appelés IPC (insulin-producing cells), chacun localisé dans un hémisphère du système nerveux [7]. Elles

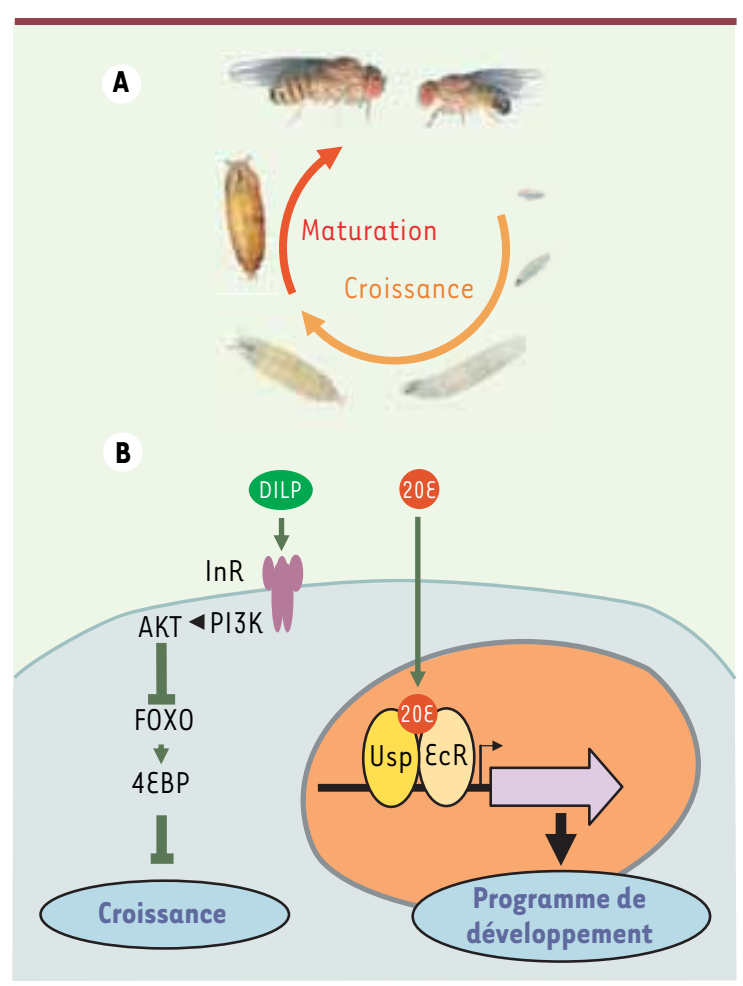

Figure 1. A. Représentation des quatre stades du cycle de vie de la drosophile : l'embryon (1 jour), la larve (4 jours), la pupe (5 jours) et l'adulte. La croissance a lieu au cours des stades larvaires sous l'influence de la voie de signalisation de l'insuline. La maturation sexuelle a ensuite lieu pendant la métamorphose. B. Les Dilp régulent la croissance cellulaire en agissant sur un récepteur unique, InR, qui active la cascade PI3-kinase/Akt et induit la rétention cytoplasmique de dFOXO et la répression transcriptionnelle de $4 \varepsilon-B P$. La durée de la période de croissance larvaire est déterminée par la $20 \varepsilon$ qui permet la transcription de gènes requis pour les processus biologiques de mues. 
agissent sur un récepteur unique, $\ln R$ (insulin receptor), qui active la cascade des protéines kinases PI3-kinase/Akt et augmente la croissance cellulaire via la rétention cytoplasmique du facteur de transcription de type forkhead dFOXO $[8,9]$ (Figure 1B). L'étude des nombreux mutants de la voie du récepteur à l'insuline a clairement établi l'importance de ce mécanisme humoral dans le contrôle de la croissance et du métabolisme des tissus larvaires en fonction des conditions environnementales [10]. Comme chez les mammifères, la production des homologues de l'insuline est régulée par les niveaux de sucres circulants $[11,12]$. Chez les insectes, le corps gras larvaire est assimilé au foie et aux tissus adipeux des vertébrés de par ses fonctions humorales et de stockage. Cet organe joue le rôle de senseur de la nutrition et contrôle la croissance des tissus périphériques en agissant à distance sur la signalisation insuline [13, 14]. Cette régulation peut alors réduire le taux de croissance et conduire à une extension compensatoire de la durée du développement afin d'atteindre une taille finale normale [15].

La durée de la période de croissance larvaire est régulée par la 20-hydroxyecdysone (20ع), la forme active de l'ecdysone. Cette hormone stéroïdienne est sécrétée par la glande en anneau larvaire, un tissu endocrinien localisé au niveau du système nerveux central. L'accumulation pulsatile de cette hormone contrôle les transitions larvaires et le début de la métamorphose [16]. De la même façon que les stéroïdes des organismes supérieurs (les corticoïdes de type cortisol ou aldostérone, et les hormones sexuelles de type œstrogènes ou androgènes), la $20 \varepsilon$ agit via des membres de la famille des récepteurs nucléaires qui se comportent comme des facteurs de transcription dont l'activité est contrôlée par le ligand [17]. La $20 \varepsilon$ permet ainsi la transcription de gènes requis pour les processus biologiques de mue (Figure 1B).

Nous avons examiné les fonctions possibles de la $20 \varepsilon$ dans le contrôle de la croissance de l'organisme en modifiant les taux de production de cette hormone [18]. Pour cela, des changements de masse de la glande en anneau ont été induits en modulant l'activité de la PI3-kinase dans ce tissu. Comme attendu, la modulation de cette voie de signalisation cause des effets de croissance autonomes dans la glande mais qui s'accompagnent, de façon surprenante, d'effets opposés sur la croissance de l'organisme. Ainsi, la diminution de taille de la glande en anneau induit un accroissement de la taille des animaux. Inversement, une augmentation de la taille de la glande induit une réduction de la taille des mouches (Figure 2A). L'ajout de $20 \varepsilon$ dans la nourriture des larves ou l'inactivation du récepteur nucléaire à l'ecdysone, respectivement, reproduisent ces effets, indiquant que le facteur responsable est l'ecdysone circulante. L'analyse des taux de transcription d'un gène cible de l'ecdysone, $\varepsilon 74 B$, montre en effet que les variations de taille de la glande en anneau s'accompagnent de variations des niveaux circulants de 20ع. De manière surprenante, les effets observés sur la croissance ne sont pas dus à un changement du programme développemental des animaux, comme conséquence possible de la modification de la fonction d'horloge de l'ecdysone. L'observation précise de la croissance des animaux démontre au contraire que l'ecdysone freine le rythme de croissance sans modifier la longueur de la période larvaire. Cela révèle une nouvelle fonction de l'ecdysone comme contre-hormone de croissance. À l'échelon cellulaire, plusieurs indicateurs tels que l'activité de la kinase Akt, la localisation du

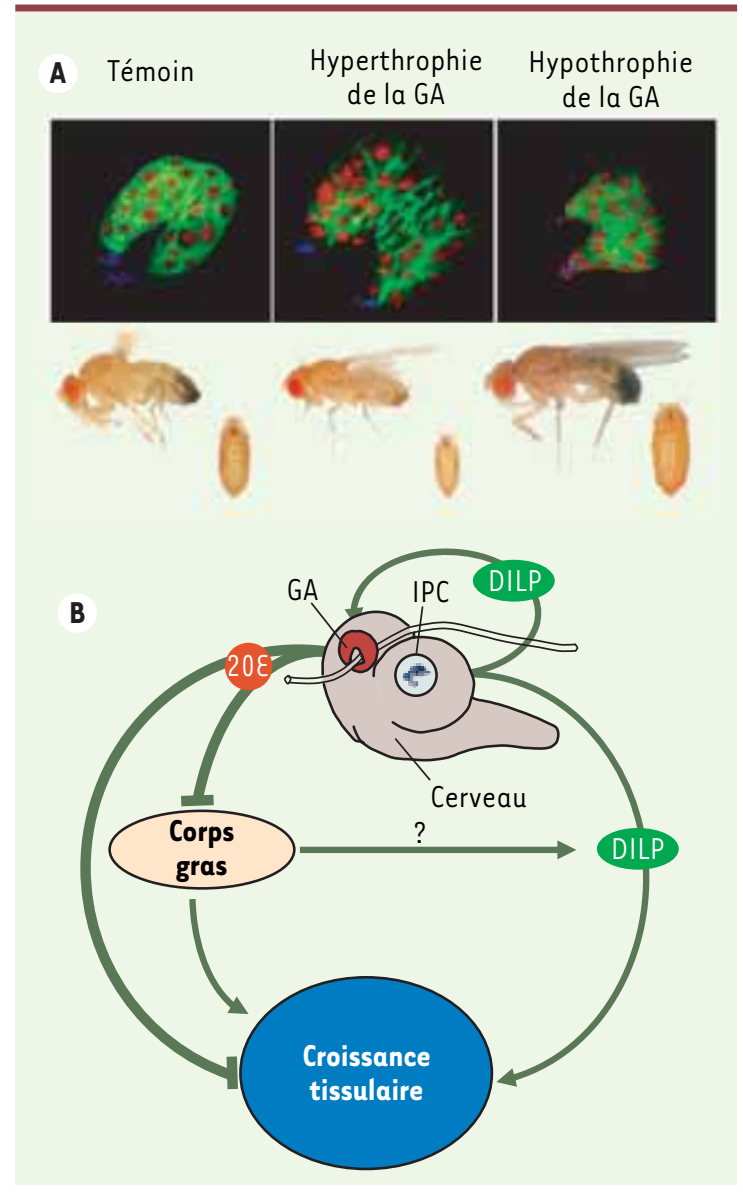

Figure 2. A. Les changements de la taille de la glande en anneau s'accompagnent d'effets opposés sur la croissance de l'organisme. Ainsi, la diminution de taille de la glande en anneau induit un accroissement de la taille des animaux. Inversement, une augmentation de la taille de la glande induit une réduction de la taille des mouches. Les contours cellulaires sont marqués en vert, les noyaux cellulaires en rouge et les cellules nerveuses en bleu. B. Modèle du contrôle de la croissance larvaire par les forces antagonistes des voies de signalisation de la $20 \varepsilon$ et des Dilp. Les Dilp sont produits par les IPC et activent la croissance. Ils peuvent aussi stimuler la synthèse de la 20ع. La $20 \varepsilon$ agit sur le corps gras et les tissus périphériques et provoque une inhibition systémique de la croissance induite par la voie de signalisation insuline. A : aorte; GA: glande en anneau; IPC: insulin-producing cells; 0 : œsophage; SNC : système nerveux central. 
facteur de transcription dFOXO, et le niveau d'expression d'une de ses cibles ( $4 \varepsilon-B P$, un inhibiteur de la synthèse protéique), montrent que l'activité de I'IIS est inversement corrélée au taux d'ecdysone circulante. En freinant le taux de croissance via dFOXO, cette hormone stéroïdienne constitue une force antagoniste à la croissance tissulaire médiée par la voie de signalisation IGF/DILP-InR-PI3K. Ces effets de l'ecdysone sont relayés en partie par les fonctions de régulation de la croissance du corps gras larvaire.

$\varepsilon$ n conclusion, cette étude révèle l'existence d'interactions complexes entre les voies de signalisation de l'insuline et de l'ecdysone pour la détermination de la taille finale de l'organisme (Figure 2B). La voie de signalisation de l'insuline stimule la production d'ecdysone dans la glande en anneau; l'ecdysone en retour inhibe l'activité de la voie de signalisation de l'insuline et la croissance des tissus larvaires, et promeut les transitions larvaires et la maturation. Ces interactions entre les deux voies permettent le couplage entre le taux de croissance et la durée du développement grâce à un effecteur commun, l'ecdysone. La signalisation insuline relayant l'information nutritionnelle dans l'organisme, ce mécanisme permet également de conserver le couplage entre la croissance et le temps de développement en condition de carence nutritionnelle. Enfin, ces résultats définissent la molécule dFOXO comme un nouveau point de convergence moléculaire entre la signalisation des récepteurs nucléaires aux hormones stérö̈diennes et la voie des IGF/insulines. Une telle interaction fonctionnelle, si elle est conservée chez I'homme, pourrait jouer un rôle important dans des pathologies cancéreuses et métaboliques où ces signalisations sont impliquées. $\diamond$

Steroids, insulin and growth:

the flies dope the research

\section{RÉFÉRENCES}

1. Leevers SJ, McNeill H. Controlling the size of organs and organisms. Curr Opin Cell Biol 2005; 17 : 604-9.

2. Johnston LA, Gallant P. Control of growth and organ size in Drosophila. Bioessays $2002 ; 24$ : 54-64.

3. Rogulja D, Irvine KD. Regulation of cell proliferation by a morphogen gradient. Cell $2005 ; 123: 449-61$.

4. Stern D. Body-size control : how an insect knows it has grown enough. Curr Biol 2003; 13 : R267-9.

5. Nijhout HF. The control of growth. Development 2003; $130: 5863-7$.
6. Garofalo RS. Genetic analysis of insulin signaling in Drosophila. Trends Endocrinol Metab2002 ; 13 : 156-62.

7. Rulifson $\varepsilon$ J, Kim SK, Nusse R. Ablation of insulinproducing neurons in flies: growth and diabetic phenotypes. Science $2002 ; 296$ : 1118-20.

8. Junger MA, Rintelen F, Stocker $\mathrm{H}$, et al. The Drosophila Forkhead transcription factor FOXO mediates the reduction in cell number associated with reduced insulin signaling. J Biol $2003 ; 2: 20$.

9. Puig 0, Marr MT, Ruhf ML, Tjian R. Control of cell number by Drosophila FOXO : downstream and feedback regulation of the insulin receptor pathway. Genes Dev 2003 ; 17 : 2006-20.

10. Hafen $\varepsilon$. Interplay between growth factor and nutrient signaling : lessons from Drosophila TOR. Curr Top Microbiol Immunol 2004 ; 279 : 153-67.

11. Ikeya HA. The regulation of Drosophila insulin like peptides gene expression in neurosecretory cells. $43^{\text {rd }}$ Annual Drosophila research conference. San Diego, 2002.

12. Kim SK, Rulifson $\varepsilon$ J. Conserved mechanisms of glucose sensing and regulation by Drosophila corpora cardiaca cells. Nature $2004 ; 431$ : 316-20.

13. Colombani J, Arquier N, Leopold P. Les mouches gardent la ligne : slimfast, le corps gras et le contrôle humoral de la croissance. Med Sci (Paris) 2004 ; $20: 141-3$.

14. Colombani J, Raisin S, Pantalacci S, et al. A nutrient sensor mechanism controls Drosophila growth. Cell 2003 ; $114: 739-49$.

15. Shingleton AW, Das J, Vinicius L, Stern DL. The temporal requirements for insulin signaling during development in Drosophila. PLoS Biol 2005 ; 3 : e289.

16. Riddiford LM. Hormone receptors and the regulation of insect metamorphosis. Receptor 1993 ; 3 : 203-9.

17. Thummel CS. Files on steroids : Drosophila metamorphosis and the mechanisms of steroid hormone action. Trends Genet 1996; 12 : 306-10.

18. Colombani J, Bianchini L, Layalle $S$, et al. Antagonistic actions of ecdysone and insulins determine final size in Drosophila. Science 2005 ; 310 : 667-70.

\section{NOUVELLE}

\section{Les protéines $\mathbf{G}$ \\ hétérotrimériques \\ au cours de la division asymétrique chez la drosophile}

Marion Ségalen, Nicolas David, Yohanns Bellaïche
Institut Curie, UMR144,

26, rue d'Ulm, 75248 Paris Cedex 05, France. yohanns.bellaiche@curie.fr
> Les métazoaires sont constitués d'un grand nombre de types cellulaires différents. Une division est dite asymétrique lorsqu'elle engendre des cellules filles d'identités différentes. Ainsi, la division asymétrique est un mode de diversification cellulaire fondamental au cours du développement
[1], notamment pour permettre le maintien de cellules souches. L'un des mécanismes de divisions asymétriques nécessite l'acquisition d'une polarité cellulaire qui se manifeste par la localisation asymétrique de molécules, appelées déterminants cellulaires. Le fuseau s'oriente ensuite pour permet- tre la ségrégation des déterminants cellulaires dans une seule des cellules filles lui conférant ainsi une identité différente de sa sœur [2]. L'étude des divisions asymétriques chez les vertébrés et les invertébrés a mis en lumière le rôle conservé des protéines $G$ hétérotrimériques au cours de ce processus. 